\title{
Comparative evaluation of the algorithms for parametric mapping of the novel myocardial PET imaging agent ${ }^{18}$ F-FPTP
}

\author{
Ji Who Kim ${ }^{1,5}$ - Seongho Seo ${ }^{1,5}$ - Hyeon Sik Kim ${ }^{2,5}$ • Dong-Yeon Kim ${ }^{2,5}$ • \\ Ho-Young Lee ${ }^{1,5} \cdot$ Keon Wook Kang ${ }^{1,3,5} \cdot$ Dong Soo Lee ${ }^{1,4,5} \cdot$ Hee-Seung Bom ${ }^{2,5}$. \\ Jung-Joon Min $^{2,5} \cdot$ Jae Sung Lee ${ }^{1,3,5}$
}

Received: 19 February 2017 / Accepted: 16 April 2017/Published online: 25 April 2017

(C) The Author(s) 2017. This article is an open access publication

\begin{abstract}
Objective $\left({ }^{18} \mathrm{~F}\right.$-fluoropentyl)triphenylphosphonium salt $\left({ }^{18} \mathrm{~F}\right.$-FPTP $)$ is a new promising myocardial PET imaging tracer. It shows high accumulation in cardiomyocytes and rapid clearance from liver. We performed compartmental analysis of ${ }^{18} \mathrm{~F}$-FPTP PET images in rat and evaluated two linear analyses: linear least-squares (LLS) and a basis function method (BFM) for generating parametric images. The minimum dynamic scan duration for kinetic analysis was also investigated and computer simulation undertaken.
\end{abstract}

Electronic supplementary material The online version of this article (doi:10.1007/s12149-017-1171-6) contains supplementary material, which is available to authorized users.

Jae Sung Lee

jaes@snu.ac.kr

Ji Who Kim

mwbd04@snu.ac.kr

1 Department of Nuclear Medicine, Seoul National University College of Medicine, Daehak-ro 101, Chongnogu, Seoul 03080, Korea

2 Department of Nuclear Medicine, Chonnam National University Hwasun Hospital, 160 Ilsimri, Hwasun 519-809, Jeonnam, Korea

3 Department of Biomedical Sciences, Seoul National University College of Medicine, Daehak-ro 101, Chongnogu, Seoul 03080, Korea

4 Department of Molecular Medicine and Biopharmaceutical Sciences, Graduate School of Convergence Science and Technology, Seoul National University, 1 Gwanak-ro, Gwanak-Gu, Seoul 08826, Korea

5 Department of Cardiology, Chonnam National University Hwasun Hospital, 160 Ilsimri, Hwasun 519-809, Jeonnam, Korea
Methods ${ }^{18}$ F-FPTP dynamic PET (18 min) and CT images were acquired from rats with myocardial infarction (MI) $(n=12)$. Regions of interest (ROIs) were on the left ventricle, normal myocardium, and MI region. Two-compartment $\left(K_{1}\right.$ and $\left.k_{2} ; 2 \mathrm{C} 2 \mathrm{P}\right)$ and three-compartment $\left(K_{1}-k_{3}\right.$; 3C3P) models with irreversible uptake were compared for goodness-of-fit. Partial volume and spillover correction terms $\left(V_{a}\right.$ and $\alpha=1-V_{a}$ ) were also incorporated. LLS and BFM were applied to ROI- and voxel-based kinetic parameter estimations. Results were compared with the standard ROI-based nonlinear least-squares (NLS) results of the corresponding compartment model. A simulation explored statistical properties of the estimation methods.

Results The 2C2P model was most suitable for describing ${ }^{18}$ F-FPTP kinetics. Average $K_{1}, k_{2}$, and $V_{a}$ values were, respectively, $6.8(\mathrm{ml} / \mathrm{min} / \mathrm{g}), 1.1\left(\mathrm{~min}^{-1}\right)$, and $0.44 \mathrm{in}$ normal myocardium and $1.4(\mathrm{ml} / \mathrm{min} / \mathrm{g}), 1.1\left(\mathrm{~min}^{-1}\right)$, and 0.32 , in MI tissue. Ten minutes of data was sufficient for the estimation. LLS and BFM estimations correlated well with NLS values for the ROI level $\left(K_{1}: y=1.06 x+0.13\right.$, $r^{2}=0.96$ and $\left.y=1.13 x+0.08, r^{2}=0.97\right)$ and voxel level $\quad\left(K_{1}: \quad y=1.22 x-0.30, \quad r^{2}=0.90 \quad\right.$ and $\left.y=1.26 x+0.00, r^{2}=0.92\right)$. Regional distribution of kinetic parametric images $\left(\alpha K_{1}, K_{1}, k_{2}, V_{\mathrm{a}}\right)$ was physiologically relevant. LLS and BFM showed more robust characteristics than NLS in the simulation.

Conclusions Fast kinetics and highly specific uptake of ${ }^{18}$ F-FPTP by myocardium enabled quantitative analysis with the 2C2P model using only the initial $10 \mathrm{~min}$ of data. LLS and BFM were feasible for estimating voxel-wise parameters. These two methods will be useful for quantitative evaluation of ${ }^{18}$ F-FPTP distribution in myocardium and in further studies with different conditions, disease models, and species. 
Keywords Quantification · Parametric image .

Simulation $\cdot$ Myocardial PET

$\begin{array}{ll}\text { Abbreviations } \\ \text { MBF } & \text { Myocardial blood flow } \\ \text { MFR } & \text { Myocardial flow reserve } \\ { }^{18} \text { F-FPTP } & \left({ }^{18} \text { F-fluoropentyl)triphenylphosphonium salt }\right. \\ \text { CAD } & \text { Coronary artery disease } \\ \text { MI } & \text { Myocardial infarction } \\ \text { LV } & \text { Left ventricular } \\ \text { ROI } & \text { Regions of interest } \\ C_{\mathrm{a}} & \text { Tracer arterial blood radioactivity } \\ & \text { concentration } \\ C_{\mathrm{T}} & \text { Activity concentration in tissue concentration } \\ K_{1}, k_{2}, k_{3} & \text { Rate constant } \\ V_{a} & \text { Blood volume fraction } \\ A & \text { Perfusible tissue fraction or recovery } \\ \text { AIC } & \text { coefficient; } 1 \text { - } V_{a} \\ \text { NLS } & \text { Akaike information criterion } \\ \text { LLS } & \text { Nonlinear least-squares method } \\ \text { BFM } & \text { Linear least-squares method } \\ \text { CV } & \text { Basis function method } \\ & \text { Coefficient of variation }\end{array}$

\section{Introduction}

Absolute quantification of regional myocardial blood flow (MBF) and myocardial flow reserve (MFR) on PET scans enables comprehensive evaluation of asymptomatic and symptomatic coronary artery disease (CAD) [1-3].

The most widely used tracers for myocardial PET scans are ${ }^{15} \mathrm{O}$-water, ${ }^{13} \mathrm{~N}$-ammonia, and ${ }^{82} \mathrm{Rb}$ which have short physical half-lives ( $2 \mathrm{~min}, 10 \mathrm{~min}$, and $76 \mathrm{~s}$, respectively) $[1,2,4,5]$. Although ${ }^{15} \mathrm{O}$-water is a physiologically ideal myocardial perfusion agent because of its high first-pass extraction fraction and metabolically inert property, its clinical use is limited because of rapid equilibrium between myocardium and the blood pool and poor image quality [2]. ${ }^{13} \mathrm{~N}$-ammonia yields markedly better image quality than ${ }^{15} \mathrm{O}$-water because ${ }^{13} \mathrm{~N}$-ammonia is a soluble substance trapped in the myocardium and it has longer half-life and shorter positron range than ${ }^{15} \mathrm{O}$ [1]. However, the requirement of an on-site cyclotron and synthesis module prevents wide clinical use of ${ }^{13} \mathrm{~N}$-ammonia $[4,6]$. In contrast, ${ }^{82} \mathrm{Rb}$ does not require an on-site cyclotron and synthesis module, and its short half-life allows rapid rest and stress imaging studies for evaluating MFR. Thus, ${ }^{82} \mathrm{Rb}$ is currently more widely used for clinical purposes $[1,4-6]$. However, ${ }^{82} \mathrm{Rb}$ PET has the limitation of relatively poor image quality because of its low extraction fraction and long positron range $[1,4,5,7,8]$. In addition, the high cost of monthly replacement of the ${ }^{82} \mathrm{Sr} /{ }^{82} \mathrm{Rb}$ generator limits the use of this tracer to large medical centers [6-9].

The development of ${ }^{18} \mathrm{~F}$-labeled myocardial PET tracers was motivated by the limitations of the existing myocardial perfusion imaging agents for PET [1, 2, 7-12]. The long half-life of ${ }^{18} \mathrm{~F}$ (110 min) allows more counts on the PET image, treadmill exercise for stress imaging, and delivery of labeled tracer to hospitals that do not have a cyclotron $[1,8,9] .{ }^{18} \mathrm{~F}$ also yields better PET spatial resolution than other radioisotopes because of its shorter positron range (i.e., seven times shorter than ${ }^{82} \mathrm{Rb}$ ) $[4,8,9]$.

$\left({ }^{18} \mathrm{~F}\right.$-fluoropentyl)triphenylphosphonium salt $\left({ }^{18} \mathrm{~F}\right.$-FPTP $)$ is a promising ${ }^{18} \mathrm{~F}$-labeled myocardial imaging agent for PET, specifically accumulating to a high degree in cardiomyocytes via a negative inner transmembrane potential of mitochondria (Fig. 1; Suppl. Figure 1) [13-15]. It was also shown that ${ }^{18}$ F-FPTP has greater first-pass myocardial extraction than ${ }^{13} \mathrm{~N}$-ammonia in isolated perfused rat heart, indicating the suitability of ${ }^{18} \mathrm{~F}$-FPTP as a quantitative myocardial PET imaging agent $[14,15]$. In addition, no metabolite was detected in the serum of mice $30 \mathrm{~min}$ after intravenous injection of ${ }^{18} \mathrm{~F}$-FPTP, implying that the metabolite correction would not be necessary in the kinetic analysis of ${ }^{18}$ F-FPTP myocardial PET data [13]. There was also a strong correlation between myocardial defect size measured by ${ }^{18}$ F-FPTP PET and the hypoperfused area measured by quantitative 2,3,5-triphenyltetrazolium chloride staining in a rat myocardial infarction (MI) model [16]. Rapid washout from the liver is another merit of ${ }^{18} \mathrm{~F}$ FPTP, which is advantageous during the evaluation of lateral myocardial wall uptake $[2,5,15,16]$.

In this study, we performed compartmental analysis on rat ${ }^{18}$ F-FPTP PET images to identify the most suitable compartment model. We then evaluated two linear analysis methods for estimating the robust kinetic parameters and generating parametric images. The minimum dynamic scan duration for kinetic analysis was also investigated. In addition, computer simulation studies were performed to confirm the two linear methods results.

\section{Materials and methods}

\section{Radiochemistry}

The radiotracer ${ }^{18}$ F-FPTP was prepared as described in a previous study [13].

\section{Animal model}

All procedures in this study were approved by the Chonnam National University Animal Research Committee and the Guide for the Care and Use of Laboratory Animals. In 
Fig. 1 Spatiotemporal distribution of ${ }^{18}$ F-FPTP. a Serial positron emission tomography (PET) scan data from a rat with myocardial infarction showing rapid accumulation of ${ }^{18}$ F-FPTP in the myocardium and the marked contrast between normal myocardium and other neighboring organs such as liver and lung. b Short-axis, vertical long-axis, and horizontal longaxis images and a polar map of ${ }^{18}$ F-FPTP PET data summed between 1 and 18 min after contrast injection

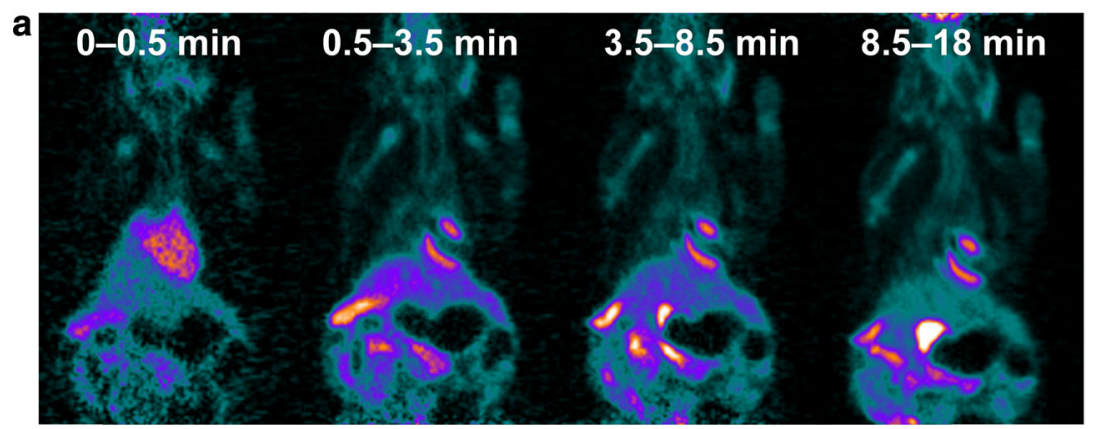

b

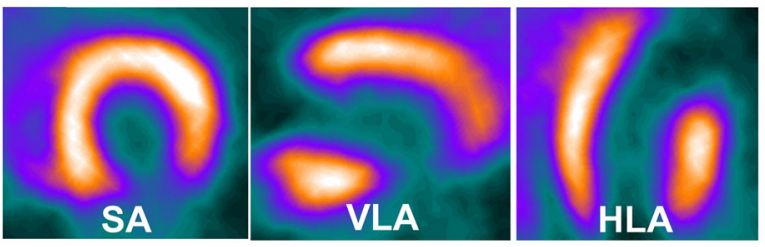

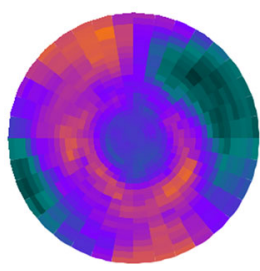

this study, we used 8-week-old male Sprague-Dawley rats ( $n=12$, weight 250-260 g). To induce MI, left coronary artery occlusion surgery was performed on the rats 1 day before the PET scans.

\section{PET/CT procedure}

Among twelve rat PET/CT data, three data acquired in our previous study [13] were included and reconstructed with our new time frame for the purposes of the current work and remaining nine data were newly acquired. In these studies, a small animal dedicated PET/CT scanner (Inveon; Siemens Medical Solutions, Knoxville, TN) was used. Dynamic PET was started with an intravenous tail-vein injection of ${ }^{18} \mathrm{~F}$-FPTP and continued for $18 \min (5 \times 1 \mathrm{~s}$, $5 \times 5 \mathrm{~s}, 3 \times 10 \mathrm{~s}, 4 \times 15 \mathrm{~s}, 16 \times 30 \mathrm{~s}, 8 \times 60 \mathrm{~s})$. PET images were reconstructed with $128 \times 128 \times 159$ matrices of $0.78 \times 0.78 \times 0.80$-mm voxel size using a filtered back-projection algorithm (Ramp filter, cutoff $=0.5)$. An $\mathrm{X}$-ray CT transmission scan was performed after the PET scan.

\section{Image analysis}

Regions of interest (ROIs) for the left ventricular (LV) cavity, normal myocardium, and MI region were drawn on static PET images generated by accumulating PET counts from 1 to $18 \mathrm{~min}$ and co-registered with the CT images of each animal using MRIcro software (http://www.mricro. com). The LV ROI to obtain the image-derived input function for kinetic analysis was drawn on the center of the LV cavity through multiple transaxial slices with the size of 6-10 voxels per slice to avoid spillover contamination from the myocardial activity. The ROI for normal myocardium was drawn on the voxels located sufficiently far from the MI and centered in the automatically drawn peak activity contour along the myocardium. The CT information was supplementary and was used for drawing ROIs on MI regions (The MI ROIs were drawn only in 10 rats because the MI was not clearly induced in 2 rats). The time-activity curves were then extracted by applying these ROIs to the serial time frames of the PET data. No metabolite correction was applied to the LV input function [13]. Time-activity curves between 0 and $10 \mathrm{~min}$ were used for the kinetic analysis.

Rat mode in PMOD software (version 3.6; PMOD Technologies, Zurich, Switzerland) was used to generate polar map images of ${ }^{18}$ F-FPTP PET. FIRE software [17] was used to reorient static PET images and fusion between parametric images.

\section{Compartment modeling: nonlinear analysis and model selection}

To determine the suitable tracer kinetic model of ${ }^{18} \mathrm{~F}$-FPTP PET, a two-compartment model with $K_{1}(\mathrm{ml} / \mathrm{min} / \mathrm{g})$ and $k_{2}$ $\left(\min ^{-1}\right)$ and a three-compartment model with irreversible uptake $\left[K_{1}, k_{2}\right.$, and $\left.k_{3}\left(\mathrm{~min}^{-1}\right)\right]$ (Suppl. Figure 2) were compared in terms of goodness-of-fit for the tissue timeactivity curves. The three-compartment model with reversible uptake was not considered because of the obvious irreversible characteristics of this tracer, as shown in the tissue time-activity curves (Fig. 2). In each compartment model tested, a blood volume fraction $\left(V_{a}\right)$ term was incorporated in the operation equation for curve fitting [18-23]. The following equation shows how the $V_{a}$ is incorporated into the two-compartment model equation:

$C_{\mathrm{T}}(t)=\left(1-V_{a}\right) K_{1} \exp \left(-k_{2} t\right) \otimes C_{a}(t)+V_{a} C_{a}(t)$ 


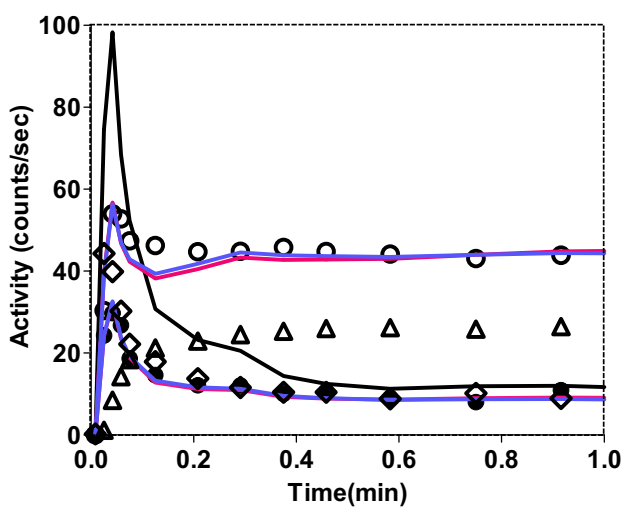

Fig. 2 Left ventricular input function (black line) and tissue timeactivity curves for a myocardial infarction (MI) region (filled circle), remote normal myocardium (open circle), liver (open triangle), and lung (open diamond) for two time scales (left 0-1 min, right

where $t$ is the time, $C_{T}(t)$ is the tissue time-activity curve, $C_{a}(t)$ is the arterial input function, and $K_{1}$ and $k_{2}$ are, respectively, the rate constants describing the uptake to and washout from the tissue compartment.

For the model selection, the tissue time-activity curves were fitted to the models using a nonlinear least-squares (NLS) method based on the Levenberg-Marquardt algorithm [19, 24]. Akaike Information Criteria (AIC) were used to determine the most appropriate model for ${ }^{18} \mathrm{~F}$-FPTP $[25,26]$.

\section{Compartment modeling: linear analysis methods and parametric image generation}

In addition to the NLS curve fitting, the kinetic parameters were estimated using linear analysis methods. These methods are less vulnerable to noise and provide much faster computation. They are useful for robust estimation of kinetic parameters and generation of their parametric images $[20,23,27,28]$. The linear analysis methods were applied only to the two-compartment model because it turned out to be more appropriate than the three-

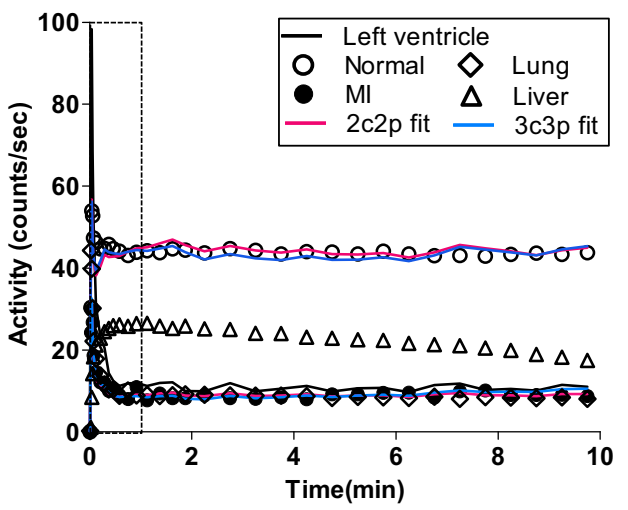

0-10 min). Fitting curves for the MI region and normal myocardium were obtained using two-compartment (red line) and three-compartment (blue line) models

compartment model for describing the kinetics of ${ }^{18} \mathrm{~F}$ FPTP (see "Results" for more detail).

We adopted two linear analysis methods. One is the multiple linear least-squares (LLS) method modified from our previous approach to the kinetic analysis of ${ }^{15} \mathrm{O}$-water [20]. The other is the basis function method (BFM), which is another well-established linear method used to generate parametric images [21, 22, 28, 29].

In addition to the ROI analysis, the LLS and BFM methods were used to generate the parametric image of each kinetic parameter through the voxel-wise parameter estimation [21, 22, 28-30] and post-Gaussian filtering with 1-mm kernel size (full width at half maximum).

\section{Simulation}

We also performed a simulation study to explore the statistical properties of the estimation methods (NLS, LLS, BFM) tested for ${ }^{18}$ F-FPTP [20, 23, 27]. Average arterial input function of ${ }^{18} \mathrm{~F}$-FPTP obtained from the rat studies was employed for the simulation study [20]. For generating simulated tissue time-activity curves, three levels of $K_{1}$ $(2.0,6.0$, and $10.0 \mathrm{ml} / \mathrm{min} / \mathrm{g})$ were assumed, and $V_{a}$ and $K_{1} /$
Table 1 Comparison of kinetic parameters estimated using twocompartment and threecompartment models and goodness-of-fit

\begin{tabular}{lllllll}
\hline Model & $K_{1}(\mathrm{ml} / \mathrm{min} / \mathrm{g})$ & $k_{2}\left(\mathrm{~min}^{-1}\right)$ & $k_{3}\left(\mathrm{~min}^{-1}\right)$ & $V_{\mathrm{a}}$ & RMSE & AIC \\
\hline \multicolumn{2}{l}{ Normal myocardium } & & & & & \\
2C & $6.8 \pm 1.8$ & $1.1 \pm 0.3$ & - & $0.44 \pm 0.10$ & 3.07 & 40.8 \\
3C & $6.9 \pm 1.8$ & $1.2 \pm 0.4$ & $0.01 \pm 0.01$ & $0.43 \pm 0.11$ & 3.06 & 42.6 \\
MI tissue & & & & & \\
2C & $1.4 \pm 0.5$ & $1.1 \pm 0.4$ & - & $0.32 \pm 0.11$ & 1.19 & 11.3 \\
3C & $1.4 \pm 0.5$ & $1.3 \pm 0.3$ & $0.02 \pm 0.02$ & $0.33 \pm 0.12$ & 1.32 & 16.7 \\
\hline
\end{tabular}

$K_{1}, k_{2}, k_{3}$ and $V_{a}$ data are means \pm standard deviations

$R M S E$ root mean square error, AIC Akaike information criteria, $2 C$ two-compartment, $3 C$ threecompartment 
$k_{2}$ were fixed at 0.2 and 3.0 , respectively, based on the results of rat studies. Poisson-like random noise generated with the consideration of activity and frame duration was added to the simulated tissue time-activity curves with six noise levels from 0 to 15 (voxel level time-activity curves in the rat studies had the noise level between 5 and 10 ) [20]. A total of 10,000 data sets were generated for each pair of $K_{1}$ and noise level [20]. Finally, the coefficient of variation $(\mathrm{CV})$, bias, and error in the estimation of each parameter were calculated [20, 27].

\section{Results}

\section{Spatiotemporal distribution of ${ }^{18}$ F-FPTP}

Figure 1a shows the serial PET scan data of a rat, revealing the dynamic whole-body distribution of ${ }^{18}$ F-FPTP. Rapid clearance of ${ }^{18} \mathrm{~F}$-FPTP from blood was observed in the LV cavity region. It had rapidly accumulated in normal myocardium and was highly sustained during the whole scan duration. The high contrast between normal and MI regions
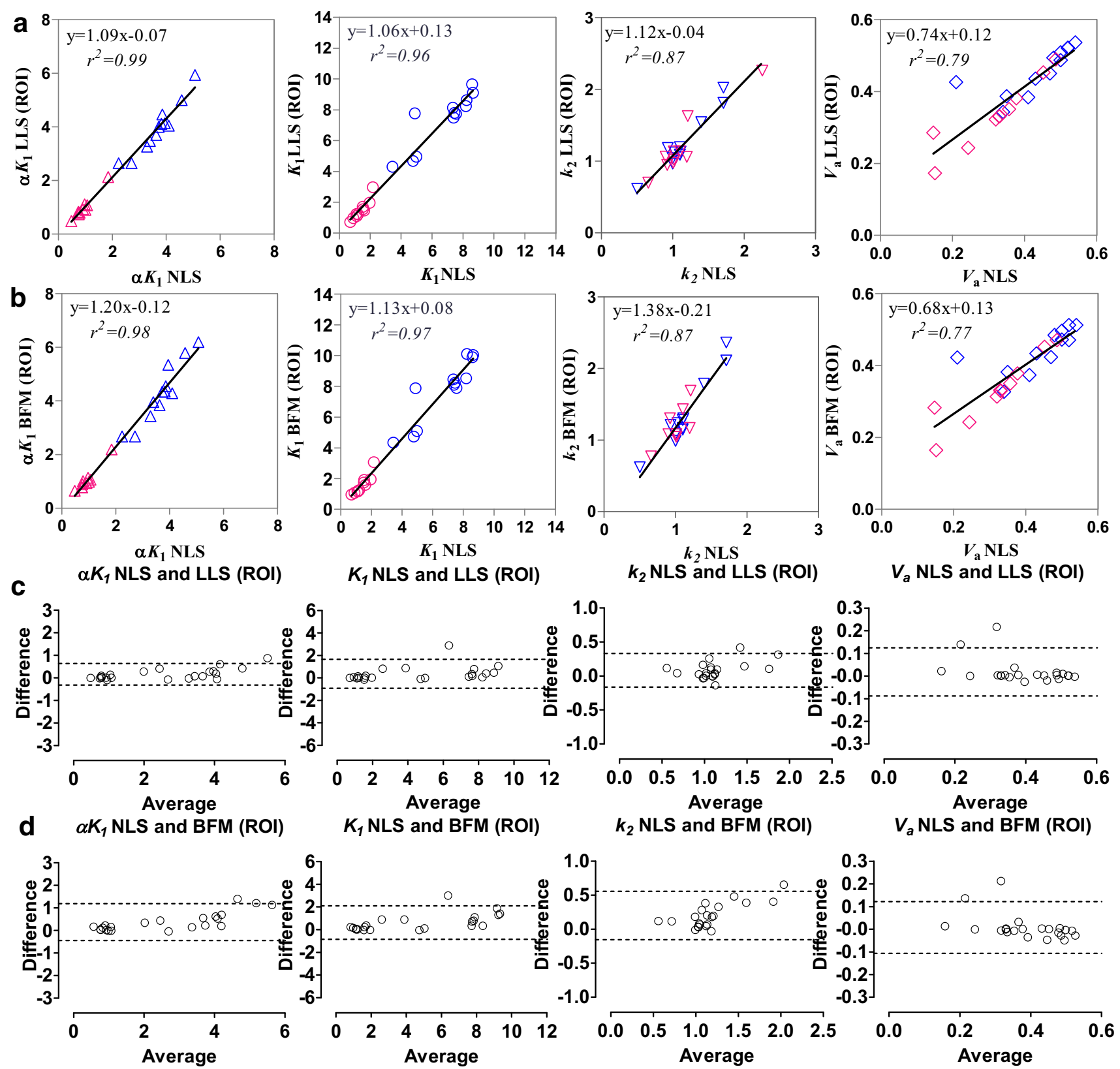

Fig. 3 Scatter plot and Bland-Altman analysis of the kinetic parameters $\left(K_{1}, k_{2}, V_{a}\right)$ obtained using two linear analysis methods-linear least-squares (LLS) and basis function method (BFM) for the two-compartment model applied to the region of interest (ROIs)

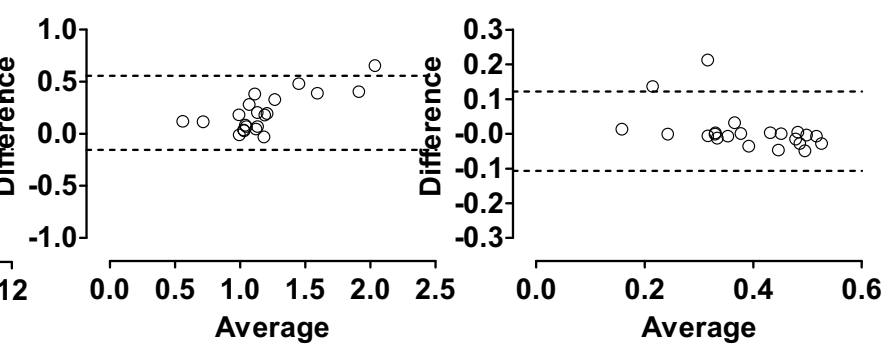

time-activity curves and those using nonlinear least-squares (NLS) estimates from ROI time-activity curves. a, c LLS. b, d BFM. In $\mathbf{a}$ and $\mathbf{b}$, different colors are used for the normal (blue) and MI (red) data 
in the myocardium was also well maintained until the end of the PET scan. ${ }^{18}$ F-FPTP uptake in the liver was quite low, making great image contrast between myocardium and liver which will be useful for mitigating the incorrect estimation of tracer uptake in the lateral wall of the heart observed with other MPI tracers, such as ${ }^{13} \mathrm{~N}$-ammonia [13].

Short-axis, vertical long-axis, and horizontal long-axis images and the polar map of ${ }^{18}$ F-FPTP PET data summed between 1 and 18 min after injection are shown in Fig. 1b. They show excellent image contrast between myocardial uptake of ${ }^{18}$ F-FPTP and the LV blood pool cavity. There is clear discrimination of the MI region in the anterolateral LV wall induced by occlusion of the left coronary artery from the myocardium with intact perfusion.

Figure 2 shows the representative LV input function and tissue time-activity curves for the MI region, remote normal myocardium, liver, and lung in two time scales (0-1 and $0-10 \mathrm{~min}$ ) in a single rat, confirming the favorable spatiotemporal properties of ${ }^{18}$ F-FPTP as a myocardial PET imaging agent (Fig. 1). Myocardium/blood activity ratios were $3.83 \pm 0.75$ (mean \pm standard deviation) at $5 \mathrm{~min}, 3.73 \pm 0.53$ at $10 \mathrm{~min}$, and $3.67 \pm 0.61$ at $15 \mathrm{~min}$. Representative activity ratio plots in a single rat are shown in Suppl. Figure 3. They indicate that ${ }^{18}$ F-FPTP quickly reaches equilibrium between the myocardium and blood in rats, which is sustained until the end of the PET scan with about a four times higher ratio in normal myocardium than in the MI region.

\section{Nonlinear analysis and model selection}

Both the two- and three-compartment model equations incorporated with $V_{a}$ yielded an excellent NLS curve fitting as shown in Fig. 2. Kinetic parameters estimated by the NLS method using the two- and three-compartment models and their AIC values are summarized in Table 1. Both models provided much lower $K_{1}$ values in the MI region relative to normal myocardium, but there were similar $k_{2}$ values between the normal and MI regions. The $K_{1}, k_{2}$, and $V_{a}$ values estimated using the two- and three-compartment models were comparable. The $k_{3}$ values estimated using the three-compartment model were negligibly small, and AIC values of the two-compartment model were lower than those of three-compartment model. Accordingly, the simpler model, the two-compartment model, was selected as the optimal model for describing ${ }^{18}$ F-FPTP kinetics, and it was employed for subsequent linear analysis methods.

\section{Linear analysis and parametric image}

The kinetic parameters obtained using the LLS and BFM methods for a two-compartment model applied to the ROI time-activity curves are correlated with the NLS estimates from ROI time-activity curves in Fig. 3. All the parameters $\left(K_{1}, k_{2}, V_{a}\right)$ estimated using LLS (Fig. 3a, c) and BFM (Fig. 3b, d) were well correlated with NLS estimates except for the low $V_{a}$ values. $K_{1}$ and $k_{2}$ estimates showed better correlation and less deviation than the $V_{a}$ values.

Figure 4 (short-axis) and Suppl. Figures 4 and 5 (vertical and horizontal long-axes) show the parametric images generated using NLS, LLS, and BFM. All three methods offered similar spatial distributions of kinetic parameters, but the two linear analysis methods (LLS and BFM) offered better image quality than NLS. The parametric images showed physiologically and physically relevant parameter distributions. The $K_{1}$ images representing the partial volume and spillover corrected uptake or perfusion showed the sharp contrast between normal myocardium and the MI region and the uniform $K_{1}$ distribution across the myocardium between the endocardial and epicardial walls. The $\alpha K_{1}\left(\alpha=1-V_{a}\right.$; perfusible tissue fraction or recovery coefficient $[18,19])$ images offered the uptake ratio distribution weighted by partial volume effect (PVE)s. The $V_{a}$ images illustrated well the blood pool distribution, particularly in the left and right ventricles, and spillover of blood activity into the myocardium. All three methods showed uniform $k_{2}$ distribution in normal myocardium. However, the NLS and BFM methods provided too high and non-zero $k_{2}$ values in both ventricles, whereas the LLS method yielded $k_{2}$ parametric images with good contrast between myocardium and ventricles.

Kinetic parameters were obtained by applying the ROIs to the parametric images and calculating the average parameter values within the ROIs, which were compared with the NLS estimates from ROI time-activity curves (Fig. 5a, c, LLS; Fig. 5b, d, BFM). The voxel-wise

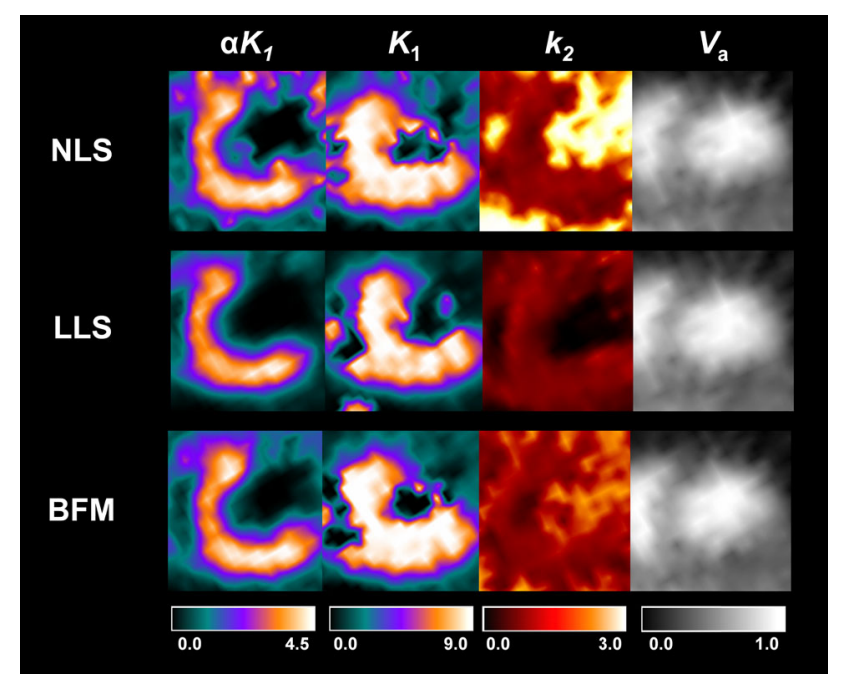

Fig. 4 Parametric images of $\alpha K_{1}\left(\alpha=1-V_{a}\right), K_{1}, k_{2}$ and $V_{a}$ generated using NLS, LLS, and BFM (short-axis) 

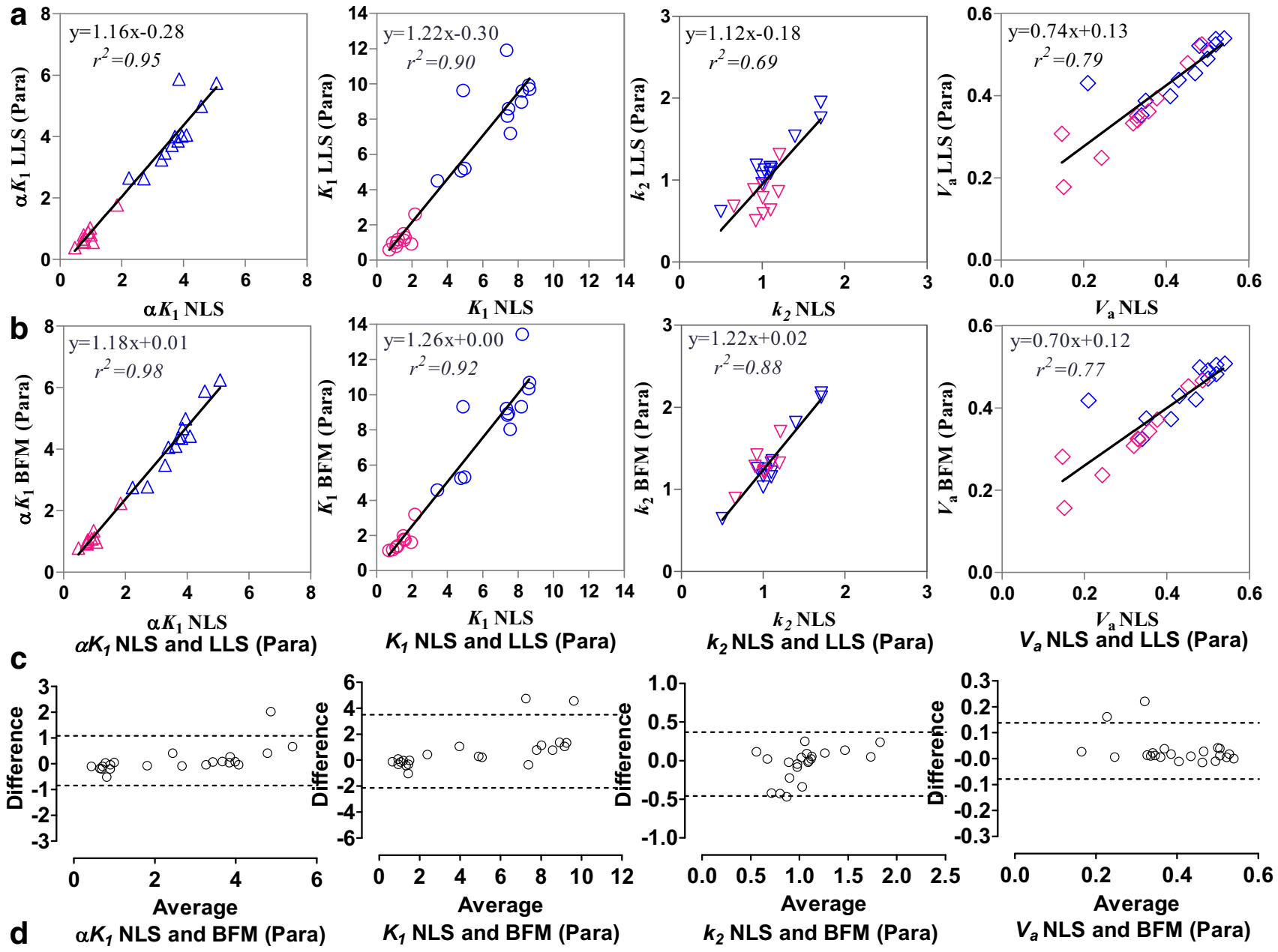

$K_{1}$ NLS and BFM (Para)
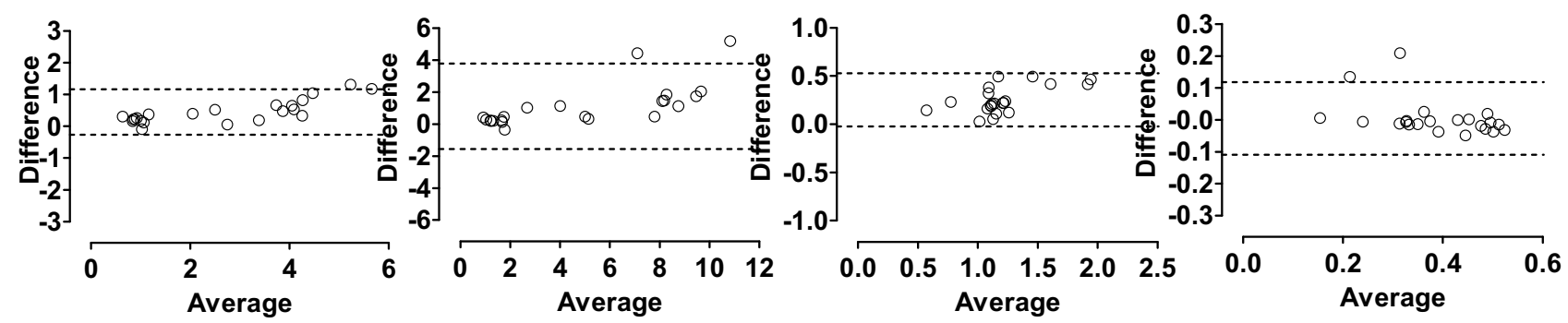

Fig. 5 Scatter plot and Bland-Altman analysis of the kinetic parameters $\left(K_{1}, k_{2}, V_{a}\right)$ obtained by applying the ROIs onto the parametric images obtained using two linear analysis methods (LLS,

BFM) and those using NLS estimates from ROI time-activity curves. a, c, LLS. b, d, BFM. In a and b, different colors are used for the normal (blue) and MI (red) data

parameter estimation resulted in relatively higher $K_{1}$ values, especially in the voxels with high $K_{1}$.

\section{Scan duration}

Figure 6 shows the kinetic parameters estimated using different scan durations, indicating that 5- and 10-min data collections are sufficient for estimating kinetic parameters using ROI- and voxel-wise estimations, respectively. The $K_{1}$ value (the parameter of interest in MBF imaging)

showed earlier convergence (even with only 3 min dynamic PET data) than other parameters in ROI analysis which is used in most clinical settings for MBF estimation.

\section{Simulation study}

Figure 7 shows the $\mathrm{CV}$ and bias in the estimation of each parameter using the NLS, LLS, and BFM methods. The LLS and BFM showed smaller CV in parameter estimation than NLS, and LLS was superior to BFM in the estimations 

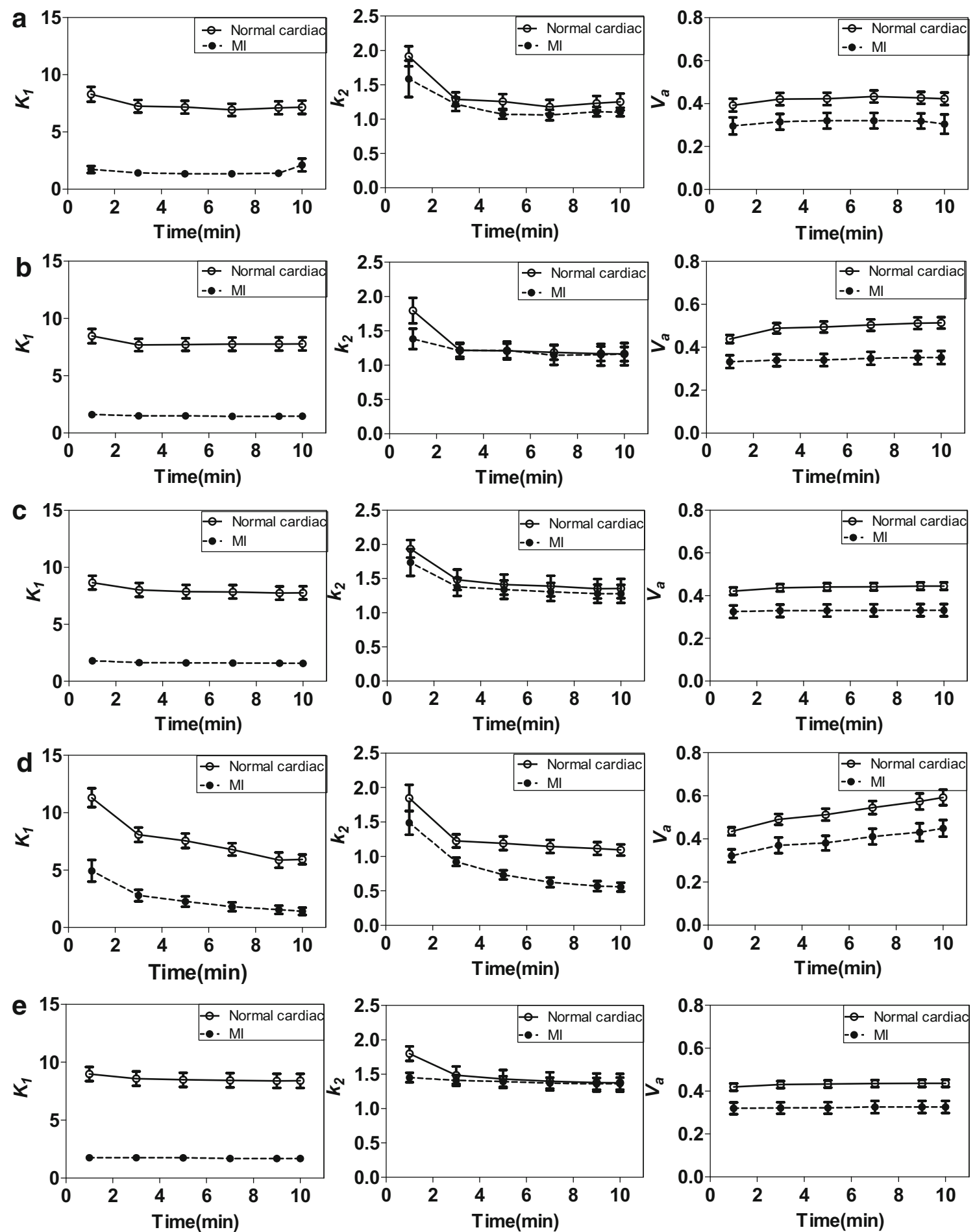

Fig. 6 Kinetic parameters estimated with different scan durations using the a NLS method on ROI time-activity curves, b LLS method on ROI time-activity curves, $\mathbf{c}$ BFM method on ROI time-activity curves, d LLS parametric images, and e BFM parametric images

of $K_{1}$ and $k_{2}$. The BFM was the best estimation method in terms of the bias, but the LLS showed relatively higher bias in a high $K_{1}$ level. Representative noisy time-activity curves for different noise levels are shown in Suppl. Figure 6.

\section{Discussion}

In this study, we performed quantitative analysis on ${ }^{18} \mathrm{~F}$ FPTP dynamic PET images from a rat model of MI to explore the spatiotemporal dynamics of this tracer, select a 

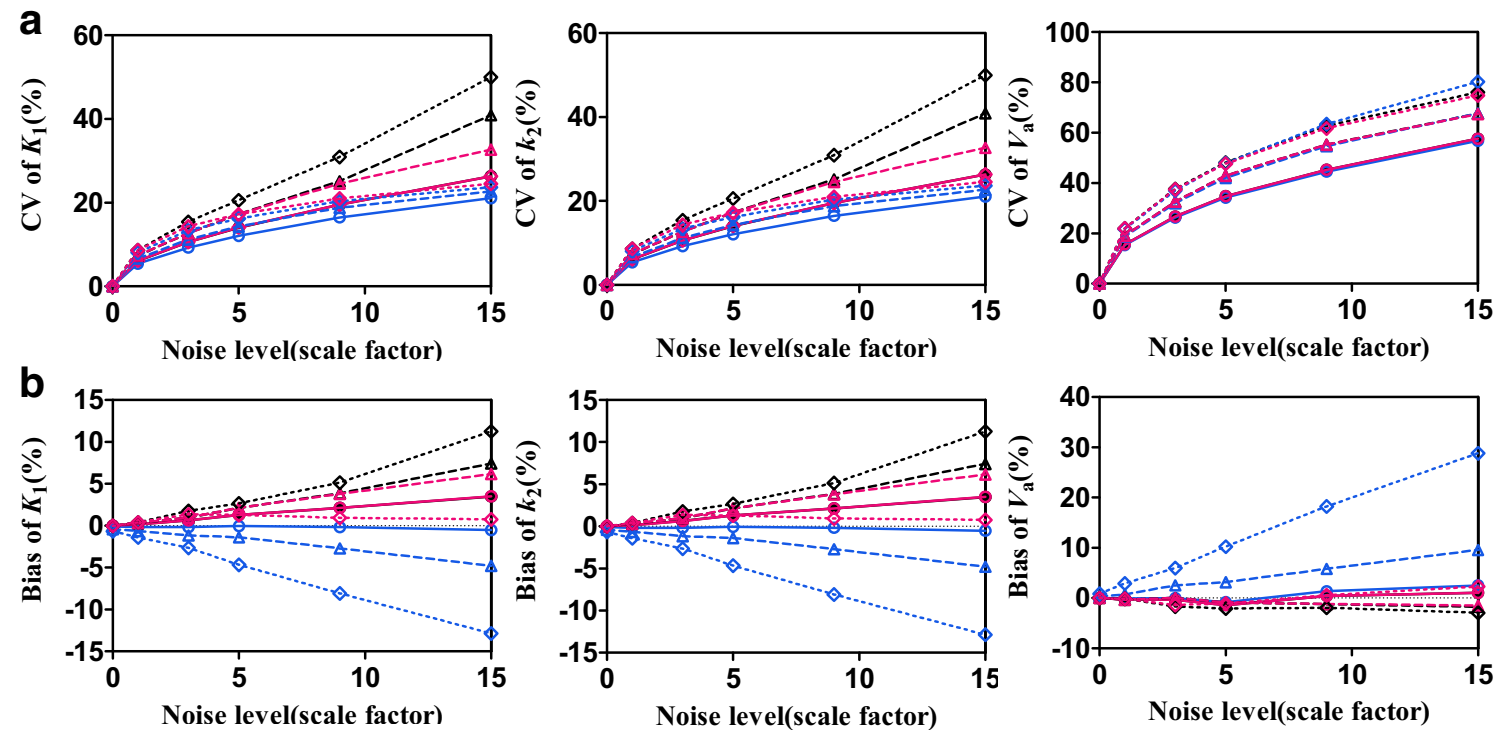

Fig. 7 Results of the simulation study: coefficients of variation (CV) (a) and bias (b) in the estimation of each parameter using the NLS (black symbols and lines), LLS (blue), and BFM (red) methods. Three $K_{1}$ levels were considered open circle 2.0, open triangle 6.0, open diamond 10.0

suitable tracer kinetic model for describing its kinetics, and investigate the properties of linear analysis methods for generating parametric images of kinetic parameters.

The model selection study for kinetic analysis suggests that the two-compartment model, which incorporates the $K_{1}, k_{2}$, and $V_{a}$ parameters, is suitable for describing the kinetics of ${ }^{18}$ F-FPTP in rat myocardium. The additional tissue compartment and kinetic parameters to distinguish the free and bound tracers in cellular space could not improve the quality of curve fitting, indicating fast equilibrium between the free and bound status of ${ }^{18} \mathrm{~F}$-FPTP. Easy and robust quantification of myocardial perfusion will be possible because kinetic modeling of ${ }^{18} \mathrm{~F}$-FPTP does not require metabolite correction and is based on the twocompartment model.

The simplicity of two-compartment model has led to the feasibility of relatively simple linear analysis methods (LLS, $\mathrm{BFM}$ ) for the noisy ROI kinetic analysis and parametric image generation. In the simulation study, the LLS and BFM showed the lowest variation and bias, respectively (Fig. 7), in agreement with the indications shown in the parametric images generated based on them (Fig. 4). The high correlation between the parameters obtained using LLS and BFM methods and those from the NLS ROI analysis, which is the gold standard but requires a long computation time, has also verified the feasibility of the linear analysis methods (Figs. 5, 7). The parametric images of ${ }^{18}$ F-FPTP provided physiologically relevant results (Fig. 4; Suppl. Figures 4 and 5). The $K_{1}$ and $\alpha K_{1}$ parametric images (flow-related images with and without partial volume correction) well accounted for the perfusion difference between normal myocardium and MI regions. Although the $K_{1}$ image provided partial volume-corrected perfusion information, the image quality was worse than that with $\alpha K_{1}$, mainly because of the division operation required for obtaining $K_{1}$ values. The distribution of $k_{2}$ was more uniform than $K_{1}$ or $\alpha K_{1}$, and $V_{a}$ images clearly showed the blood pool distribution in the heart. However, only the LLS showed good contrast between myocardium and the LV cavity in $k_{2}$ parametric images, which may be caused by the better parameter estimation performance of lower parameter values than attained with the other methods.

In this study, input function was obtained from LV pool of PET images. Although the kinetic model itself includes PVE, the input function is assumed to be free from the PVE as in our previous rat PET study [31]. However, the PVE on the input function is not avoidable sometimes even in the small animal dedicated PET scanners. Previously we have shown that LV input function in mice PET studies is underestimated by $5-10 \%$ relative to the arterial blood samples [32]. However, in this study, we used more advanced small animal PET scanner than the previous study (Inveon vs. Focus 120) [33-35]. In addition, rats have much larger LV cavity than mice, causing less severe PVE. In case there is the underestimation of LV input function due to the PVE, it would more influence on the $K_{1}$ and $V_{a}$ (overestimation) rather than $k_{2}$.

Usually, a weighting factor is considered for compensating the different signal-to-noise ratios (SNRs) among PET time frames because the different SNRs cause bias in parameter estimation. However, the weighting did not prove effective in this study for improving the bias properties and parametric image quality (Suppl. Figure 7; frame duration was used as the weighting factor). The weight would be more effective for the radiotracers with binding and dissociation as 
parameters of interest and the relatively short half-life of the radioisotope [27]. However, it seems that the slope of the early frames with rapid buildup and the ratio of tissue and blood activity in the later frames are the most dominant factors when determining the kinetic parameters of ${ }^{18} \mathrm{~F}$ FPTP. The parameters determined mainly in each of these early and later portions of the time-activity curves are influenced very little by the weighting factors.

\section{Conclusion}

A novel, promising, myocardial PET imaging agent, ${ }^{18} \mathrm{~F}$ FPTP, was quantitatively evaluated in rats with MI. For describing the kinetics of this tracer, a two-compartment model was more suitable than a three-compartment model. Two linear analyses for parametric image generation showed that the kinetic parameters were well correlated with nonlinearly estimated gold standard ones and yielded the high quality images of those parameters. The first 10 min of the dynamic scans was sufficient for obtaining accurate kinetic parameters in quantitative PET studies. Finally, the two algorithms for parametric mapping of ${ }^{18} \mathrm{~F}$ FPTP in myocardium will be useful for further studies under different conditions, disease model, and species.

Acknowledgements This article was supported by Grants from the Korea Healthcare Technology R\&D Project, Ministry of Health and Welfare (HI13C0163020015) and Ministry of Science, ICT and Future Planning (NRF-2014M3C7A1046042).

\section{Compliance with ethical standards}

Conflict of interest The authors have no conflicts to disclose.

Open Access This article is distributed under the terms of the Creative Commons Attribution 4.0 International License (http://creative commons.org/licenses/by/4.0/), which permits unrestricted use, distribution, and reproduction in any medium, provided you give appropriate credit to the original author(s) and the source, provide a link to the Creative Commons license, and indicate if changes were made.

\section{Appendix}

In the LLS method, the measured arterial input function and tissue time-activity curve are related to the ideal ones, as shown in the following equations, by incorporating partial-volume and spillover effects [9, 12, 18-23]:

$$
\begin{aligned}
& \tilde{C}_{a}(t)=C_{a}(t) \\
& \tilde{C}_{T}(t)=\left(1-V_{a}\right) C_{T}(t)+V_{a} C_{a}(t)
\end{aligned}
$$

The ideal input function and tissue time-activity curve expressed with the measured curves are then obtained by transposing the above equations and substituting them into the differential equation for an ideal tissue time-activity curve $\left(\mathrm{d} C_{T} / \mathrm{d} t=K_{1} C_{a}-k_{2} C_{T}\right)$, yielding the following equation [20]:

$$
\begin{aligned}
\frac{\mathrm{d}}{\mathrm{d} t}\left\{\tilde{C}_{T}(t)-V_{a} \tilde{C}_{a}(t)\right\}= & \left(1-V_{a}\right) K_{1} \tilde{C}_{a}(t) \\
& -k_{2}\left\{\tilde{C}_{T}(t)-V_{a} \tilde{C}_{a}(t)\right\} .
\end{aligned}
$$

By rearranging this equation and applying integration to both sides, the following linear equation in the form of $\boldsymbol{y}=\boldsymbol{X} \boldsymbol{\theta}$ is derived:

$$
\begin{aligned}
\boldsymbol{y} & =\left[\tilde{C}_{T}(t)\right] \\
\boldsymbol{X} & =\left[\tilde{C}_{a}(t) \int \tilde{C}_{a}(t) \mathrm{d} t \int \tilde{C}_{T}(t) \mathrm{d} t\right] \\
\boldsymbol{\theta} & =\left[V_{a}\left(1-V_{a}\right) K_{1}+k_{2} V_{a}-k_{2}\right]^{\boldsymbol{T}}
\end{aligned}
$$

Then, an estimated $\boldsymbol{\theta}$ based on LLS criteria was given by the $\boldsymbol{\theta}=\left(\boldsymbol{X}^{T} \boldsymbol{X}\right)^{-1} \boldsymbol{X}^{T} \boldsymbol{y}$, and the LLS solution of $\left(K_{1}, k_{2}, V_{a}\right)$ is analytically calculated from the $\boldsymbol{\theta}$.

For applying BFM, a set of linear models was derived from Eq. 1 as follows:

$C_{\mathrm{T}}(t)=\left(1-V_{a}\right) K_{1} B_{j}(t)+V_{a} C_{a}(t)$ for $1 \leq j \leq m$

where each $B_{j}(t)=\exp \left(k_{2, j} \cdot t\right) \otimes C_{a}(t)$ is a basis function, $k_{2, j}$ is the $j$ th value in a predefined discrete pool of $k_{2}$ values, and $m$ is the number of the predefined $k_{2}$ values. We assumed that $k_{2}$ is bounded between 0.01 and $2.5\left(\mathrm{~min}^{-1}\right)$ based on the estimated $k_{2}$ values using the NLS method. Then, 100 basis functions with discrete $k_{2}$ values spaced logarithmically between the bounds were generated, and an LLS estimate of $\left(K_{1}, V_{a}\right)$ was obtained for each basis function. Finally, the BFM solution of $\left(K_{1}, k_{2}, V_{a}\right)$ was determined as the one with the smallest sum of squared errors between measured and estimated tissue time-activity curves.

\section{References}

1. Schindler TH, Schelbert HR, Quercioli A, Dilsizian V. Cardiac PET imaging for the detection and monitoring of coronary artery disease and microvascular health. JACC Cardiovasc Imaging. 2010;3:623-40.

2. Salerno M, Beller GA. Noninvasive assessment of myocardial perfusion. Circ Cardiovasc Imaging. 2009;2:412-24.

3. Parkash R, deKemp RA, Ruddy TD, Kitsikis A, Hart R, Beauchesne L, et al. Potential utility of rubidium 82 PET quantification in patients with 3 -vessel coronary artery disease. J Nucl Cardiol. 2004;11:440-9.

4. Al-Mallah MH, Sitek A, Moore SC, Di Carli M, Dorbala S. Assessment of myocardial perfusion and function with PET and PET/CT. J Nucl Cardiol. 2010;17:498-513.

5. Anagnostopoulos C, Georgakopoulos A, Pianou N, Nekolla SG. Assessment of myocardial perfusion and viability by positron emission tomography. Int J Cardiol. 2013;167:1737-49.

6. Machac J. Radiopharmaceuticals for clinical cardiac PET imaging. In: Di Carli MF, Lipton MK, editors. Cardiac PET and PET/ CT Imaging. New York: Springer; 2007. p. 73-82. 
7. Dilsizian V, Taillefer R. Journey in evolution of nuclear cardiology: will there be another quantum leap with the F-18-labeled myocardial perfusion tracers? JACC Cardiovasc Imaging. 2012;5:1269-84.

8. Maddahi J. Properties of an ideal PET perfusion tracer: new PET tracer cases and data. J Nucl Cardiol. 2012;19:S30-7.

9. Packard RR, Huang SC, Dahlbom M, Czernin J, Maddahi J. Absolute quantitation of myocardial blood flow in human subjects with or without myocardial ischemia using dynamic flurpiridaz F 18 PET. J Nucl Med. 2014;55:1438-44.

10. Shoup TM, Elmaleh DR, Brownell AL, Zhu A, Guerrero JL, Fischman AJ. Evaluation of (4-[18F]Fluorophenyl)triphenylphosphonium ion. A potential myocardial blood flow agent for PET. Mol Imaging Biol. 2011;13:511-7.

11. Gurm GS, Danik SB, Shoup TM, Weise S, Takahashi K, Laferrier $\mathrm{S}$, et al. 4-[18F]-tetraphenylphosphonium as a PET tracer for myocardial mitochondrial membrane potential. JACC Cardiovasc Imaging. 2012;5:285-92.

12. Nekolla SG, Reder S, Saraste A, Higuchi T, Dzewas G, Preissel A, et al. Evaluation of the novel myocardial perfusion positronemission tomography tracer ${ }^{18} \mathrm{~F}-\mathrm{BMS}-747158-02$ : comparison to ${ }^{13} \mathrm{~N}$-ammonia and validation with microspheres in a pig model. Circulation. 2009; 119:2333-42.

13. Kim DY, Kim HS, Le UN, Jiang SN, Kim HJ, Lee KC, et al. Evaluation of a mitochondrial voltage sensor, $\left({ }^{18} \mathrm{~F}\right.$-fluoropentyl)triphenylphosphonium cation, in a rat myocardial infarction model. J Nucl Med. 2012;53:1779-85.

14. Kim DY, Kim HS, Reder S, Zheng JH, Herz M, Higuchi T, et al. Comparison of ${ }^{18} \mathrm{~F}$-labeled fluoroalkylphosphonium cations with ${ }^{13} \mathrm{~N}^{-\mathrm{NH}_{3}}$ for pet myocardial perfusion imaging. J Nucl Med. 2015;56:1581-6.

15. Brunken RC. Promising New Fluorine-18 Labeled Tracers for PET Myocardial Perfusion Imaging. J Nucl Med. 2015;56:1478-9.

16. Kim DY, Kim HS, Jang HY, Kim JH, Bom HS, Min JJ. Comparison of the cardiac microPET images obtained using $\left[^{18-}\right.$ F]FPTP and $\left[{ }^{13} \mathrm{~N} \mathrm{NH}_{3}\right.$ in rat myocardial infarction models. ACS Med Chem Lett. 2014;5:1124-8.

17. Lee JS, Park KS, Lee DS, Lee CW, Chung JK, Lee MC. Development and applications of a software for functional image registration (FIRE). Comput Methods Programs Biomed. 2005;78:157-64.

18. Iida H, Rhodes CG, de Silva R, Yamamoto Y, Araujo LI, Maseri A, et al. Myocardial tissue fraction-correction for partial volume effects and measure of tissue viability. $J$ Nucl Med. 1991;32:2169-75.

19. Muzik O, Beanlands RS, Hutchins GD, Mangner TJ, Nguyen N, Schwaiger M. Validation of nitrogen-13-ammonia tracer kinetic model for quantification of myocardial blood flow using PET. J Nucl Med. 1993;34:83-91.

20. Lee JS, Lee DS, Ahn JY, Yeo JS, Cheon GJ, Kim SK, et al. Generation of parametric image of regional myocardial blood flow using $\mathrm{H}_{2}^{15} \mathrm{O}$ dynamic PET and a linear least-squares method. J Nucl Med. 2005;46:1687-95.

21. Lodge MA, Carson RE, Carrasquillo JA, Whatley M, Libutti SK, Bacharach SL. Parametric images of blood flow in oncology PET studies using $\left[{ }^{15} \mathrm{O}\right]$ water. J Nucl Med. 2000;41:1784-92.
22. Boellaard R, Knaapen P, Rijbroek A, Luurtsema GJ, Lammertsma AA. Evaluation of basis function and linear least squares methods for generating parametric blood flow images using ${ }^{15} \mathrm{O}$ water and positron emission tomography. Mol Imaging Biol. 2005;7:273-85.

23. Chen K, Lawson M, Reiman E, Cooper A, Feng D, Huang SC, et al. Generalized linear least squares method for fast generation of myocardial blood flow parametric images with $\mathrm{N}-13$ ammonia PET. IEEE Trans Med Imaging. 1998;17:236-43.

24. Moré JJ. The Levenberg-Marquardt algorithm: implementation and theory. In: Watson GA, editor. Numerical Analysis. BerlinHeidelberg: Springer; 1978. p. 105-16.

25. Yamaoka K, Nakagawa T, Uno T. Application of akaike's information criterion (AIC) in the evaluation of linear pharmacokinetic equations. J Pharmacokinet Biopharm. 1978;6:165-75.

26. Ludden TM, Beal SL, Sheiner LB. Comparison of the Akaike Information Criterion, the Schwarz criterion and the $\mathrm{F}$ test as guides to model selection. $\mathrm{J}$ Pharmacokinet Biop. 1994;22:431-45.

27. Feng D, Wang Z, Huang SC. A study on statistically reliable and computationally efficient algorithms for generating local cerebral blood flow parametric images with positron emission tomography. IEEE Trans Med Imaging. 1993;12:182-8.

28. Gunn RN, Lammertsma AA, Hume SP, Cunningham VJ. Parametric imaging of ligand-receptor binding in PET using a simplified reference region model. Neuroimage. 1997;6:279-87.

29. Watabe H, Jino H, Kawachi N, Teramoto N, Hayashi T, Ohta Y, et al. Parametric imaging of myocardial blood flow with ${ }^{15} \mathrm{O}$ water and PET using the basis function method. J Nucl Med. 2005;46:1219-24.

30. Lee JS, Lee DS, Ahn JY, Cheon GJ, Kim SK, Yeo JS, et al. Blind separation of cardiac components and extraction of input function from $\mathrm{H}_{2}^{15} \mathrm{O}$ dynamic myocardial PET using independent component analysis. J Nucl Med. 2001;42:938-43.

31. Kim JH, Kim YH, Kim YJ, Yang BY, Jeong JM, Youn H, et al. Quantitative positron emission tomography imaging of angiogenesis in rats with forelimb ischemia using ${ }^{68}$ Ga-NOTAc(RGDyK). Angiogenesis. 2013;16:837-46.

32. Kim SJ, Lee JS, Im KC, Kim SY, Park SA, Lee SJ, et al. Kinetic modeling of $3^{\prime}$-deoxy $-3^{\prime}-{ }^{18} \mathrm{~F}$-fluorothymidine for quantitative cell proliferation imaging in subcutaneous tumor models in mice. J Nucl Med. 2008;49:2057-66.

33. Bao Q, Newport D, Chen M, Stout DB, Chatziioannou AF. Performance evaluation of the Inveon dedicated PET preclinical tomograph based on the NEMA NU-4 standards. J Nucl Med. 2009;50:401-8.

34. Kim JS, Lee JS, Im KC, Kim SJ, Kim SY, Lee DS, et al. Performance measurement of the microPET focus 120 scanner. J Nucl Med. 2007;48:1527-35.

35. Goertzen AL, Bao Q, Bergeron M, Blankemeyer E, Blinder S, Cañadas M, et al. NEMA NU 4-2008 comparison of preclinical PET imaging systems. J Nucl Med. 2012;53:1300-9. 